\title{
A SIMPLE PROOF OF KOORNWINDER'S ADDITION FORMULA FOR THE LITTLE $q$-LEGENDRE POLYNOMIALS
}

\author{
MIZAN RAHMAN
}

(Communicated by Kenneth R. Meyer)

\begin{abstract}
Recently Koornwinder found an addition formula for the little $q$ Legendre polynomials by using Masuda et al.'s result that they are related to the matrix elements of the irreducible unitary representation of the twisted $\mathrm{SU}(2)$ quantum group. Here we give an alternate derivation of the addition formula by using some summation and transformation formulas of basic hypergeometric series.
\end{abstract}

\section{INTRODUCTION}

The little $q$-Jacobi polynomials are defined in [1] by the formula

$$
p_{n}(x ; a, b \mid q)={ }_{2} \phi_{1}\left(q^{-n}, a b q^{n+1} ; a q ; q, x q\right), \quad n=0,1, \ldots,
$$

where the expression on the right side is a special case of the basic hypergeometric series

$$
{ }_{r+1} \phi_{r}\left[\begin{array}{cc}
a_{1}, a_{2}, \ldots, a_{r+1} \\
b_{1}, \ldots, b_{r}
\end{array} ; q, z\right]=\sum_{k=1}^{\infty} \frac{\left(a_{1}, a_{2}, \ldots, a_{r+1} ; q\right)_{k}}{\left(q, b_{1}, \ldots, b_{r} ; q\right)_{k}} z^{k},
$$

with

$$
\left(a_{1}, a_{2}, \ldots, a_{m} ; q\right)_{k}=\left(a_{1} ; q\right)_{k}\left(a_{2} ; q\right)_{k} \cdots\left(a_{m} ; q\right)_{k},
$$

and $q$-shifted factorials defined by

$$
(a ; q)_{k}= \begin{cases}1, & \text { if } k=0, \\ (1-a)(1-a q) \cdots\left(1-a q^{k+1}\right), & \text { if } k=1,2, \ldots\end{cases}
$$

In (1.1) the two upper parameters are $q^{-n}$ and $a b q^{n+1}$, the lower parameter is $a q$, and the argument of the series is $x q$.

Recently it was shown by Masuda et al. [5] that the matrix elements of the irreducible unitary representations of the quantum group $\mathrm{S}_{\mu} \mathrm{U}(2)$ (see Woronowicz [6], [7]) can be expressed in terms of the little $q$-Jacobi polynomials. Use

Received by the editors December 2, 1988.

1980 Mathematics Subject Classification (1985 Revision). Primary 33A65.

This work was partially supported by NSERC Grant \#A6197. 
of this result enabled Koornwinder [4] to derive the following addition formula for the little $q$-Legendre polynomials:

$$
\begin{aligned}
& p_{n}\left(q^{z} ; 1,1 \mid q\right) W_{y}\left(q^{z} ; q^{x} \mid q\right) \\
&= p_{n}\left(q^{x+y} ; 1,1 \mid q\right) p_{n}=\left(q^{y} ; 1,1 / q\right) W_{y}\left(q^{z} ; q^{x} \mid q\right) \\
&+\sum_{k=1}^{n} \frac{(q ; q)_{x+y+k}(q ; q)_{n-k} q^{k(y-n+k)}}{(q ; q)_{x+y}(q ; q)_{n-k}(q ; q)_{k}^{2}} p_{n-k}\left(q^{x+y} ; q^{k}, q^{k} \mid q\right) \\
& \cdot p_{n-k}\left(q^{y} ; q^{k}, q^{k} \mid q\right) W_{y+k}\left(q^{z} ; q^{x} \mid q\right) \\
&+ \sum_{k=1}^{n} \frac{(q ; q)_{y}(q ; q)_{n+k} q^{k(x+y-n+1)}}{(q ; q)_{y-k}(q ; q)_{n-k}(q ; q)_{k}^{2}} p_{n-k}\left(q^{x+y-k} ; q^{k}, q^{k} \mid q\right) \\
& \cdot p_{n-k}\left(q^{y-k} ; q^{k}, q^{k} \mid q\right) W_{y-k}\left(q^{z} ; q^{x} \mid q\right),
\end{aligned}
$$

where

$$
W_{n}(x ; a \mid q)=p_{n}(x ; a, 0 \mid q)
$$

are the Wall polynomials (see, for instance, Chihara [2]) that satisfy the orthogonality relation

$$
\sum_{k=0}^{\infty} \frac{a^{k}}{(q ; q)_{k}} W_{m}\left(q^{k} ; a \mid q\right) W_{n}\left(q^{k} ; a \mid q\right)=(a ; q)_{\infty}^{-1} \frac{(q ; q)_{n} a^{n}}{(a ; q)_{n}} \delta_{m, n},
$$

subject to the restriction that $0<q<1$ and $|a|<1$, which we shall assume to be true throughout this paper.

The purpose of this note is to give an alternative proof of (1.5) without the use of group theory, based instead only on hypergeometric series manipulations.

To this end we shall first derive in $\S 2$ the following product formula

$$
\begin{aligned}
& p_{n}\left(q^{x} ; b, b \mid q\right) p_{n}\left(q^{y} ; b, b \mid q\right) \\
& =\sum_{j=0}^{n} \frac{\left(q^{-n}, b^{2} q^{n+1}, q^{-x} ; q\right)_{j}\left(q b^{2} ; q\right)_{2 j}}{\left(q, b q, b q, q b^{2} ; q\right)_{j}}(-1)^{j} q^{(x+y+1) j-\left(\begin{array}{l}
j \\
2
\end{array}\right)} \\
& { }_{3} \phi_{2}\left[\begin{array}{cccc}
q^{-j}, & q^{-j} / b, & q^{x-y-j} / b & \\
& q^{1+x-j}, & q^{-2 j} / b^{2} & ; q, q
\end{array}\right],
\end{aligned}
$$

where $0 \leq x \leq y$ and $x, y=0,1,2, \ldots$. By a sequence of transformations we then show in $\S 3$ that

$$
\begin{aligned}
& p_{n}\left(q^{y} ; 1,1 \mid q\right) p_{n}\left(q^{x+y} ; 1,1 \mid q\right) \\
&=\frac{\left(q^{x+1} ; q\right)_{\infty}\left(q^{x+1} ; q\right)_{y}}{(q ; q)_{y}} q^{-(x+1) y} \\
& \cdot \sum_{z=0}^{\infty} \frac{q^{(x+1) z}}{(q ; q)_{z}} W_{y}^{2}\left(q^{z} ; q^{x} \mid q\right) p_{n}\left(q^{z} ; 1,1 \mid q\right),
\end{aligned}
$$


which suggests an expansion of the form:

$$
\begin{aligned}
p_{n}\left(q^{z} ; 1,1 \mid q\right) W_{y}\left(q^{z} ; q^{x} \mid q\right) \\
\quad=\sum_{k=-n}^{n} A_{n, k}(x, y \mid q) W_{y+k}\left(q^{z} ; q^{x} \mid q\right)
\end{aligned}
$$

for the little $q$-Legendre polynomials. By using the orthogonality relation (1.7) we compute the coefficients $A_{n, k}(x, y \mid q)$ in $\S 4$ and thus complete the proof of (1.5).

\section{THE PROOF OF (1.8)}

Using [3, (8.1.3) and (8.1.4)] we find that

$$
\begin{aligned}
{ }_{2} \phi_{1}\left(q^{-n}, b^{2} q^{n+1} ; b q ; q, s q\right)_{2} \phi_{1}\left(q^{-n}, b^{2} q^{n+1} ; b q ; q, t q\right) \\
=\sum_{m=0}^{n}\left(q^{-n}, b^{2} q^{n+1} ; q\right)_{m} \sum_{k=0}^{m} \frac{t^{k} q^{k^{2}+k-m k}}{(q, b q ; q)_{k}(q, b q ; q)_{m-k}} \\
\quad \cdot \sum_{j=0}^{k} \frac{\left(q^{-k}, b^{2} q^{m+1} ; q\right)_{j}}{\left(q, b q^{m-k+1} ; q\right)_{j}}(s q)^{m-k+j} .
\end{aligned}
$$

By a straightforward series manipulation it follows that

$$
\begin{aligned}
p_{n}(s ; b, b \mid q) p_{n}(t ; b, b \mid q) & \\
= & \sum_{k=0}^{n} \frac{\left(q^{-n}, b^{2} q^{n+1} ; q\right)_{k}}{(q, b q ; q)_{k}}(s q)^{k} \sum_{j=0}^{k} \frac{\left(q^{-k}, b^{2} q^{k+1} ; q\right)_{j}}{(q, b q ; q)_{j}}(t q)^{j} \\
& \cdot{ }_{2} \phi_{1}\left(q^{j-k}, q^{-k} / b ; b q^{j+1} ; q, b t q^{k+1} / s\right),
\end{aligned}
$$

where we assume, for the sake of definiteness, that $0<t \leq s$. For $|b| \leq 1$, we can now apply Heine's transformation formula [3, (1.4.5)] to get

$$
\begin{aligned}
{ }_{2} \phi_{1}\left(q^{j-k}, q^{-k} / b ; b q^{j+1} ; q, b t q^{k+1} / s\right) \\
\quad=\frac{\left(b^{2} q^{k+j+1}, t q / s ; q\right)_{\infty}}{\left(b q^{j+1}, b t q^{k+1} / s ; q\right)_{\infty}}{ }_{2} \phi_{1}\left(q^{-k} / b, t q^{-k} / b s ; t q / s ; q, b^{2} q^{k+j+1}\right) .
\end{aligned}
$$

Substituting this into (2.2) and summing over $j$ by the $q$-binomial formula $[3,(1.3 .2)]$ we obtain

$$
\begin{aligned}
p_{n}(s ; b, b \mid q) p_{n}(t ; b, b \mid q) & \\
= & \frac{\left(q b^{2}, t q / s ; q\right)_{\infty}}{(b q, b t q / s ; q)_{\infty}} \sum_{k=0}^{n} \frac{\left(q^{-n}, b^{2} q^{n+1}, b t q / s, t^{-1} ; q\right)_{k}}{\left(q, b q, q b^{2}, q\right)_{k}} \\
& \cdot(-s t q)^{k} q_{3}^{-\left(\begin{array}{c}
k \\
2
\end{array}\right)} \phi_{2}\left[\begin{array}{ccc}
t q, & t q^{-k} / b s, & q^{-k} / b \\
t q / s, & t q^{1-k} & ; q, b^{2} q^{k+1}
\end{array}\right] .
\end{aligned}
$$


Using Hall's formula [3, (3.2.7)] and then Sears' formula [3, (3.2.5)] we find that

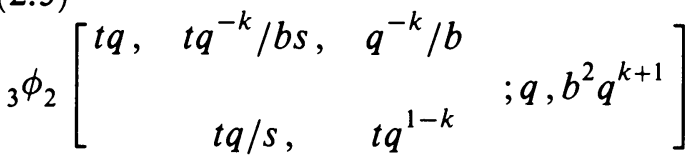

$$
\begin{aligned}
& =\frac{\left(b q, b t q^{k+1} / s ; q\right)_{\infty}}{\left(b^{2} q^{k+1}, t q / s ; q\right)_{\infty}} \phi_{2}\left[\begin{array}{cccc}
q^{-k}, & q^{-k} / b, & b s q & \\
& b q, & t q^{1-k} & ; q, b t q^{k+1} / s
\end{array}\right] \\
& =\frac{(b q, b t q / s ; q)_{\infty}}{\left(q b^{2}, t q / s ; q\right)_{\infty}} \frac{\left(q b^{2} ; q\right)_{2 k}}{(b q, b t q / s ; q)_{k}}{ }_{3} \phi_{2}\left[\begin{array}{ccc}
q^{-k}, & q^{-k} / b, & t q^{-k} / b s \\
& & \\
& t q^{1-k}, & q^{-2 k} / b^{2}
\end{array}\right] \text {. }
\end{aligned}
$$

Thus we have the product formula

$$
\begin{aligned}
p_{n}(s ; b, b \mid q) p_{n}(t ; b, b \mid q) & \\
= & \sum_{k=0}^{n} \frac{\left(q^{-n}, b^{2} q^{n+1}, t^{-1} ; q\right)_{k}\left(q b^{2} ; q\right)_{2 k}}{\left(q, b q, b q, q b^{2} ; q\right)_{k}}(-s t q)^{k} q^{-\left(\begin{array}{l}
k \\
2
\end{array}\right)} \\
& \left.\cdot{ }_{3} \phi_{2}\left[\begin{array}{rrr}
q^{-k}, & q^{-k} / b, & t q^{-k} / b s \\
t q^{1-k}, & q^{-2 k} / b^{2}
\end{array}\right] ; q, q\right] .
\end{aligned}
$$

Replacing $s$ and $t$ by $q^{y}$ and $q^{x}$, respectively, we obtain (1.8).

\section{Proof of (1.9)}

We now set $b=1$ in (1.8), replace $y$ by $x+y$ and then interchange $x$ and $y$ to get

$$
\begin{aligned}
& p_{n}\left(q^{y} ; 1,1 \mid q\right) p_{n}\left(q^{x+y} ; 1,1 \mid q\right) \\
& =\sum_{j=0}^{n} \frac{\left(q^{-n}, q^{n+1}, q^{-y} ; q\right)_{j}(q ; q)_{2 j}}{(q, q, q, q ; q)_{j}}(-1)^{j} q^{(x+2 y+1) j-\left(\begin{array}{l}
j \\
2
\end{array}\right)} \\
& \cdot{ }_{3} \phi_{2}\left[\begin{array}{cccc}
q^{-j}, & q^{-j}, & q^{-x-j} & \\
& q^{1+y-j}, & q^{-2 j} & ; q, q
\end{array}\right] .
\end{aligned}
$$


Use of the transformation formula [3, (3.2.3)] now gives

$$
{ }_{3} \phi_{2}\left[\begin{array}{cccc}
q^{-j}, & q^{-j}, & q^{-x-j} & \\
& q^{1+y-j}, & q^{-2 j} & ; q, q
\end{array}\right]
$$

$$
\begin{aligned}
& =\frac{\left(q^{x+y+1-j} ; q\right)_{j}}{\left(q^{-2 j} ; q\right)_{j}} q^{-(x+y+1+j) j}{ }_{3} \phi_{2}\left[\begin{array}{cccc}
q^{-j}, & q^{y+1}, & q^{x+y+1} & \\
& q^{x+y+1-j}, & q^{1+y-j} & ; q, q]
\end{array}\right] \\
& =\frac{\left(q, q^{-x-y} ; q\right)_{j}}{(q ; q)_{2 j}}{ }_{3} \phi_{2}\left[\begin{array}{cccc}
q^{-j}, & q^{y+1}, & q^{x+y+1} & \\
& q^{x+y+1-j}, & q^{1+y-j} &
\end{array}\right] \text {. }
\end{aligned}
$$

Thus we have

$$
\begin{aligned}
p_{n}\left(q^{y} ; 1,1 \mid q\right) p_{n}\left(q^{x+y} ; 1,1 \mid q\right) & \\
= & \sum_{j=0}^{n} \frac{\left(q^{-n}, q^{n+1}, q^{-y}, q^{-x-y} ; q\right)_{j}}{(q, q, q ; q)_{j}}(-1)^{j} q^{(x+2 y+1) j-\left(\begin{array}{l}
j \\
2
\end{array}\right)} \\
& \cdot{ }_{3} \phi_{2}\left[\begin{array}{rrr}
q^{-j}, & q^{y+1}, & q^{x+y+1} \\
& q^{x+y+1-j}, & q^{1+y-j}
\end{array}\right] .
\end{aligned}
$$

Now, it can be shown that

(3.4)

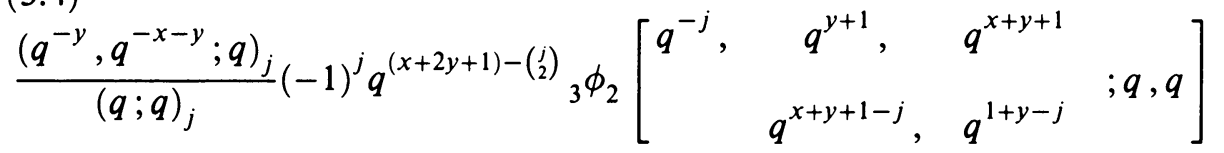

$$
\begin{aligned}
& =\frac{\left(q^{x+1} ; q\right)_{y}}{(q ; q)_{y}} q^{-(x+1) y+j} \sum_{k=0}^{y} \frac{\left(q^{-y} ; q\right)_{k}\left(q^{x+1} ; q\right)_{j+k}}{\left(q, q^{x+1} ; q\right)_{k}} q^{k} \frac{\left(q^{-j-k} ; q\right)_{y}}{\left(q^{x+1} ; q\right)_{y}} q^{(x+1+j+k) y} \\
& =\frac{\left(q^{x+1} ; q\right)_{y}}{(q ; q)_{y}} q^{j-(x+1) y} \sum_{k=0}^{y} \frac{\left(q^{-y}, q\right)_{k}\left(q^{x+1} ; q\right)_{j+k}}{\left(q, q^{x+1} ; q\right)_{k}} q^{k} \\
& \text { - }{ }_{2} \phi_{1}\left(q^{-y}, q^{x+1+j+k} ; q^{x+1} ; q, q\right) \text {, }
\end{aligned}
$$

where the last line follows by use of the $q$-Vandermode formula [3, (1.5.3)]. Substituting (3.4) into (3.3) we obtain

$$
\begin{aligned}
& p_{n}\left(q^{y} ; 1,1 \mid q\right) p_{n}\left(q^{x+y} ; 1,1 \mid q\right) \\
&=\frac{\left(q^{x+1} ; q\right)_{y} q^{-(x+1) y}}{(q ; q)_{y}} \sum_{j=0}^{n} \frac{\left(q^{-n}, q^{n+1} ; q\right)_{j}}{(q, q ; q)_{j}} q^{j} \sum_{k=0}^{y} \frac{\left(q^{-y} ; q\right)_{k} q^{k}}{\left(q, q^{x+1} ; q\right)_{k}}\left(q^{x+1} ; q\right)_{j+k} \\
& \cdot \sum_{i=0}^{y} \frac{\left(q^{-y}, q^{x+1+j+k} ; q\right)_{i}}{\left(q, q^{x+1}, q\right)_{i}} q^{i}
\end{aligned}
$$


(3.5)

$$
\begin{aligned}
= & \frac{\left(q^{x+1} ; q\right)_{\infty}\left(q^{x+1} ; q\right)_{y}}{(q ; q)_{y}} q^{-(x+1) y} \sum_{j=0}^{n} \frac{\left(q^{-n}, q^{n+1} ; q\right)_{j}}{(q, q ; q)_{j}} \\
& \cdot \sum_{k=0}^{y} \frac{\left(q^{-y} ; q\right)_{k} q^{k}}{\left(q, q^{x+1} ; q\right)_{k}} \sum_{i=0}^{y} \frac{\left(q^{-y} ; q\right)_{i} q^{i}}{\left(q, q^{x+1} ; q\right)_{i}} \sum_{z=0}^{\infty} \frac{q^{(x+1+i+j+k) z}}{(q ; q)_{z}} \\
= & \frac{\left(q^{x+1} ; q\right)_{\infty}\left(q^{x+1} ; q\right)_{y}}{(q ; q)_{y}} q^{-(x+1) y} \sum_{z=0}^{\infty} \frac{q^{(x+1) z}}{(q ; q)_{z}} W_{y}^{2}\left(q^{z} ; q^{x} \mid q\right) p_{n}\left(q^{z} ; 1,1 \mid q\right),
\end{aligned}
$$

which completes the proof of (1.9).

\section{Proof of the AdDition formula (1.5)}

From (1.7) and (1.9) it is clear that

$$
A_{n, 0}(x, y \mid q)=p_{n}\left(q^{y} ; 1,1 \mid q\right) p_{n}\left(q^{x+y} ; 1,1 \mid q\right),
$$

and

$$
\begin{aligned}
A_{n, k}(x, y \mid q)= & \frac{\left(q^{x+1} ; q\right)_{\infty}\left(q^{x+1} ; q\right)_{y+k}}{(q ; q)_{y+k}} q^{-(x+1)(y+k)} \\
& \cdot \sum_{z=0}^{\infty} \frac{q^{(x+1) z}}{(q ; q)_{z}} W_{y}\left(q^{z} ; q^{x} \mid q\right) W_{y+k}\left(q^{z} ; q^{x} \mid q\right) p_{n}\left(q^{z} ; 1,1 \mid q\right)
\end{aligned}
$$

for $|k|=1,2, \ldots, \min (n, y)$.

Suppose that $k$ is a positive integer. Then, by the $q$-binomial and the $q$ Vandermonde formulas, we have

$$
\begin{aligned}
& A_{n, k}(x, y \mid q) \\
& =\frac{\left(q^{x+1} ; q\right)_{y+k}}{(q ; q)_{y+k}} q^{-(x+1)(y+k)} \sum_{i=0}^{n} \frac{\left(q^{-n}, q^{n+1} ; q\right)_{i}}{(q, q ; q)_{i}} q^{i} \\
& \cdot \sum_{j=0}^{y} \frac{\left(q^{-y} ; q\right)_{j}\left(q^{x+1} ; q\right)_{i+j}}{\left(q, q^{x+1} ; q\right)_{j}} q^{j} \frac{\left(q^{-i-j} ; q\right)_{y+k}}{\left(q^{x+1} ; q\right)_{y+k}} q^{(x+1+i+j)(y+k)} \\
& \left.=(-1)^{y+k} q^{(y+k+1}\right)\left(q^{x+1} ; q\right)_{y+k} \\
& \cdot \sum_{i=0}^{n} \frac{\left(q^{-n}, q^{n+1} ; q\right)_{i}\left(q^{-y} ; q\right)_{y-i+k}}{(q, q ; q)_{i}\left(q, q^{x+1} ; q\right)_{y-i+k}} \\
& { }_{3} \phi_{2}\left[\begin{array}{cccc}
q^{k-i}, & q^{x+y+k+1}, & q^{y+k+1} & \\
& q^{y+k+1-i}, & q^{x+y+1+k-i} & ; q, q
\end{array}\right] .
\end{aligned}
$$


Since $\left(q^{-y} ; q\right)_{y-i+k}$ clearly vanishes unless $i \geq k$ we replace $i$ by $k+i$ in the above series and obtain after some simplification

$$
\begin{aligned}
A_{n, k}(x, y \mid q) & \frac{(q ; q)_{x+y+k}(q ; q)_{n+k}}{(q ; q)_{x+y}(q ; q)_{n-k}(q ; q)_{k}^{2}} q^{k(k+y-n)} \\
& \cdot \sum_{i=0}^{n-k} \frac{\left(q^{k-n}, q^{n+k+1}, q^{-y}, q^{-x-y} ; q\right)_{i}}{\left(q, q^{k+1}, q^{k+1} ; q\right)_{i}}(-1)^{i} q^{(x+2 y+1) i-\left(\begin{array}{l}
i \\
2
\end{array}\right)} \\
& \cdot{ }_{3} \phi_{2}\left[\begin{array}{ccc}
q^{-i}, & q^{x+y+k+1}, & q^{y+k+1} \\
q^{y+1-i}, & q^{x+y+1-i}
\end{array}\right] .
\end{aligned}
$$

By [3, (3.23)], we have

$$
\begin{aligned}
& { }_{3} \phi_{2}\left[\begin{array}{cccc}
q^{-i}, & q^{x+y+k+1}, & q^{y+k+1} & \\
& q^{y+1-i}, & q^{x+y+1-i} & ; q, q
\end{array}\right]
\end{aligned}
$$

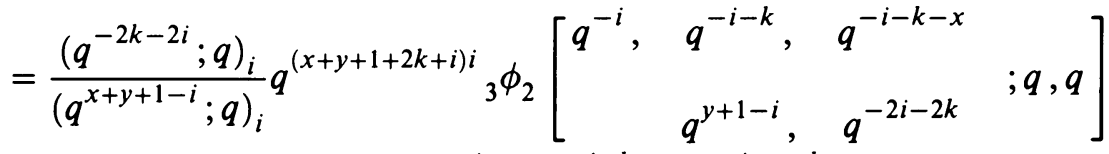

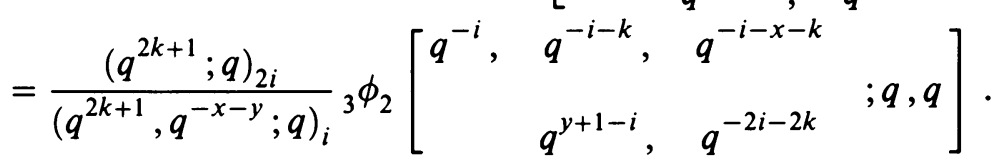

Substituting (4.5) into (4.4) we obtain

$$
\begin{aligned}
& A_{n, k}(x, y \mid q) \\
& =\frac{(q ; q)_{x+y+k}(q ; q)_{n+k}}{(q ; q)_{x+y}(q ; q)_{n-k}(q ; q)_{k}^{2}} q^{k(k+y-n)} \\
& \cdot \sum_{i=0}^{n-k} \frac{\left(q^{k-n}, q^{n+k+1}, q^{-y} ; q\right)_{i}\left(q^{2 k+1} ; q\right)_{2 i}}{\left(q, q^{k+1}, q^{k+1}, q^{2 k+1} ; q\right)_{i}}(-1)^{i} q^{(x+2 y+1)-\left(\begin{array}{l}
i \\
2
\end{array}\right)} \\
& \cdot{ }_{3} \phi_{2}\left[\begin{array}{llll}
q^{-i}, & q^{-i-k}, & q^{-i-x-k} & \\
& q^{y+1-i}, & q^{-2 i-2 k} &
\end{array}\right] \\
& =\frac{(q ; q)_{x+y+k}(q ; q)_{n+k}}{(q ; q)_{x+y}(q ; q)_{n-k}(q ; q)_{k}^{2}} q^{k(k+y-n)} \\
& \text { - } p_{n-k}\left(q^{x+y} ; q^{k}, q^{k} \mid q\right) p_{n-k}\left(q^{y} ; q^{k}, q^{k} \mid q\right) \text {, }
\end{aligned}
$$


by (2.6). To compute $A_{n, k}$ for negative $k$ 's, we note that

$$
\begin{aligned}
A_{n,-k}(x, y \mid q) & \\
= & \frac{\left(q^{x+1} ; q\right)_{y-k}}{(q ; q)_{y-k}} q^{-(x+1)(y-k)} \sum_{k=0}^{n} \frac{\left(q^{-n}, q^{n+1} ; q\right)_{i}}{(q, q ; q)_{i}} q^{i} \\
& \cdot \sum_{j=0}^{y-k} \frac{\left(q^{k-y} ; q\right)_{j}\left(q^{x+1} ; q\right)_{i+j}}{\left(q, q^{x+1} ; q\right)_{j}} q^{j} \frac{\left(q^{-i-j} ; q\right)_{y}}{\left(q^{x+1} ; q\right)_{y}} q^{(x+1+i+j) y} \\
= & (-1)^{y} q^{(y+1)+k(x+1)} \frac{(q ; q)_{y}\left(q^{x+1} ; q\right)_{y-k}}{(q ; q)_{y-k}} \\
& \cdot \sum_{i=0}^{n} \frac{\left(q^{-n}, q^{n+1} ; q\right)_{i}\left(q^{k-y}, q\right)_{y-i}}{(q, q ; q)_{i}\left(q, q^{x+1} ; q\right)_{y-i}} \\
& { }_{3} \phi_{2}\left[\begin{array}{c}
q^{k-i}, \quad q^{x+y+1}, \quad q^{y+1} \\
q^{y+1-i}, \quad q^{x+y+1-i}
\end{array}\right] .
\end{aligned}
$$

Since $\left(q^{k-y} ; q\right)_{y-i}$ vanishes unless $i \geq k$ we replace $i$ by $i+k$ and simplify to obtain

$$
\begin{aligned}
& A_{n-k}(x, y \mid q) \\
& =\frac{(q ; q)_{y}(q ; q)_{n+k} q^{k(x+y+1-n)}}{(q ; q)_{y-k}(q ; q)_{n-k}(q ; q)_{k}^{2}} \sum_{i=0}^{n-k} \frac{\left(q^{k-n}, q^{n+k+1}, q^{k-y}, q^{k-x-y} ; q\right)_{i}}{\left(q, q^{k+1}, q^{k+1} ; q\right)_{i}}
\end{aligned}
$$

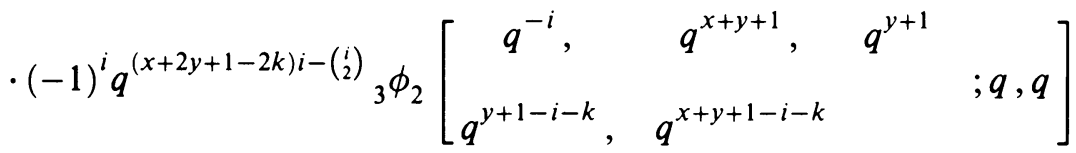

$$
\begin{aligned}
& =\frac{(q ; q)_{y}(q ; q)_{n+k} q^{k(x+y+1-n)}}{(q ; q)_{y-k}(q ; q)_{n-k}(q ; q)_{k}^{2}} \sum_{i=0}^{n-k} \frac{\left(q^{k-n}, q^{n+k+1}, q^{k-y} ; q\right)_{i}\left(q^{2 k+i} ; q\right)_{2 i}}{\left(q, q^{k+1}, q^{k+1}, q^{2 k+1} ; q\right)_{i}} \\
& \cdot(-1)^{i} q^{(x+2 y+1-2 k) i-\left(\begin{array}{l}
i \\
2
\end{array}\right)}{ }_{3} \phi_{2}\left[\begin{array}{llll}
q^{-i}, & q^{-i-k}, & q^{-x-i-k} & \\
& q^{y+1-k-i}, & q^{-2 i-2 k} & ; q, q
\end{array}\right] \text {, }
\end{aligned}
$$

by $[3,(3.2 .3)]$. It is now clear from (2.6) that

(4.9)

$$
\begin{aligned}
& A_{n,-k}(x, y \mid q) \\
& \quad=\frac{(q ; q)_{y}(q ; q)_{n+k} q^{k(x+y+1-n)}}{(q ; q)_{y-k}(q ; q)_{n-k}(q ; q)_{k}^{2}} p_{n-k}\left(q^{y-k} ; q^{k}, q^{k} \mid q\right) p_{n-k}\left(q^{x+y-k} ; q^{k}, q^{k} \mid q\right) .
\end{aligned}
$$

The addition formula (1.5) then follows from (1.10), (4.1), (4.6) and (4.9). 


\section{REFERENCES}

1. G. E. Andrews and R. Askey, Enumeration of partitions: The role of Eulerian series and $q$ orthogonal polynomials, pp. 3-26 in Higher Combinatorics, ed. M. Aigner, Reidel, 1977.

2. T. S. Chihara, An introduction to orthogonal polynomials, Gordon and Breach, 1978.

3. G. Gasper and M. Rahman, Basic hypergeometric series, Cambridge University Press, (to appear).

4. T. H. Koornwinder, The addition formula for little $q$-Legendre polynomials and the twisted $\mathrm{SU}(2)$ quantum group, (to appear).

5. T. Masuda, K. Minachi, V. Nakagami, M. Noumi, and K. Ueno, Representations of quantum groups and a q-analogue of orthogonal polynomials, C. R. Acad. Sci. Paris, Sér. I Math. 307 (1988), 559-564.

6. S. L. Woronowicz, Compact matrix pseudogroups, Comm. Math. Phys. 111 (1987), 613-665.

7. __ Twisted SU(2) group. An example of a noncommutative differential calculus, Publ. Res. Inst. Math. Sci. 23 (1987), 117-181.

Department of Mathematics and Statistics, Carleton University, Ottawa, Ontario, CANADA K1S 5B6 